\title{
Management of Separated Instruments in Apical Third of Extremely Curved Canals: A Case Report
}

\author{
Muhammad Zubair Ahmad* \\ Restorative Dental Sciences Department, Alrass Dental College, Qassim University, Buraydha, Saudi Arabia \\ *Corresponding author: m.muhammad@qu.edu.sa
}

\begin{abstract}
This report describes the case of a 23 years old male patient who presented with the chief complaint of pain in mandibular right lower first molar tooth. Detailed clinical and radiographic examination revealed acute pulpitis in lower right second premolar and first molar teeth. During root canal instrumentation, a rotary instrument separated at the apical third of mesiobuccal canal. After careful assessment, it was decided to bypass the instrument and obturate the root canal space with warm vertical compaction using thermoplasticized gutta percha and resin based sealer materials. After considering the curvature of canal, thin root of tooth and location of broken instrument in the apical third of canal space, attempts of instrument removal were ruled out as a treatment option for the patient. Bypassing the broken instrument fragment with pre-curved hand files of small sizes was chosen as a predictable treatment option since it is not associated with removal of excessive remaining dentin from the roots of the teeth. Hence, teeth do not become weak and risk of post-operative root fracture is minimized. Due to successful bypassing and preparation of root canal space beyond broken instrument, canal was shaped, cleaned and disinfected adequately. Decision of instrument bypassing or removal should be taken with extreme care. Bypassing the instrument in apical third of root canal space with thin rooted teeth is the most conservative and practical approach, and objectives of adequate shaping and cleaning of canals can be achieved successfully without compromising remaining tooth structure.
\end{abstract}

\section{Keywords: separated instrument, instrument bypassing, resin based sealer, curved canals}

Cite This Article: Muhammad Zubair Ahmad, "Management of Separated Instruments in Apical Third of Extremely Curved Canals: A Case Report.” International Journal of Dental Sciences and Research, vol. 6, no. 1 (2018): 18-21. doi: 10.12691/ijdsr-6-1-4.

\section{Introduction}

Root canal treatment is considered as the one of fundamental dental therapies and is dependent upon root canal space preparation and disinfection. The most frequently involved tooth in endodontic treatment is considered to be the mandibular first molar, because of its earliest eruption among permanent teeth [1]. A separated intracanal endodontic instrument is one of the most troublesome incident during root canal preparation procedures. The prevalence of instrument separation is reported from 0.5 to $5 \%[2,3,4,5]$. Overall prognosis of treatment might be affected if the broken instrument has impeded adequate cleaning of the canal beyond the obstruction. Therefore, available scientific literature advocates for leaving of broken fragments and separated instruments in situ in some cases [4,6]. Nickel-titanium instruments are reported to be stronger than the stainless steel files but separation of these instruments can occur because of rotational stresses imposed on engine-driven instruments [7]. The ultimate objective of management of separated instrument is mainly to preserve the integrity of the root canal space and the tooth. If separated instrument is located deep in the canal and attempts of its removal are with risks of potential damaging and weakening of tooth then bypassing the instrument should be considered [8]. In this case, successful endodontic treatment with bypassing of a broken rotary Ni-Ti instrument in apical third of extremely curved mesiobuccal canal of mandibular first molar tooth is demonstrated.

\section{Case Report}

A 23 years old male patient was referred to the Department of Endodontics for endodontic treatment of his right first mandibular molar tooth. His medical history was noncontributory and clinical examination revealed mild pain on palpation and tenderness to percussion.

The preoperative radiograph showed mesial proximal carious decay involving the pulp chamber on mandibular first molar and distal proximal decay on second premolar involving the pulp chamber (Figure 1). A complete history and careful clinical and radiographic examination along with pulp sensibility tests confirmed the diagnosis of acute pulpitis in mandibular right second premolar and first molar teeth. Decision of performing endodontic treatment on both teeth was made.

After achieving anaesthesia, the whole mandibular right quadrant was isolated using a rubber dam (Hygienic Corporation, OH 44310, USA) and the access cavities prepared using Endo access and Endo-z burs (Dentsply, 
Maillefer, Baillaigues, Switzerland). Orifices of root canal system were first located with the aid of an endodontic explorer (Odous, Belo Horizonte, MG, Brazil) and then with size 10 K-files (Dentsply, Maillefer, Baillaigues, Switzerland). In molar tooth three root canals were detected (one $\mathrm{C}$ shaped canal in the distal root and two canals in the mesial root). In premolar tooth one central oval shaped canal was detected. Pulp extirpation was done using a barbed broach. Working lengths of all canals were established for both teeth (Figure 2). Canals were not found dry and there was pus discharge so iodoform containing intracanal calcium hydroxide Metapex ${ }^{\circledR}$ paste (Meta Biomed Co., Ltd, Korea)) was placed as intracanal medicament, canal orifices were secured using sterile cotton pallets and access cavities were sealed using zinc oxide/ calcium sulfate (Cavit, 3M ESPE, St. Paul, MN, USA) as temporary coronal restoration. Another appointment was given after one week.

Patient did not visit at his given appointment because of relief of pain. He visited back after 1 year because of flare up. During this time, he visited a private general dentist who extracted his premolar tooth because of extensive carious decay onto the root surface and advised him to consult his treating endodontist to complete root canal therapy in first molar tooth.

Another periapical radiograph was taken before start of the procedure (Figure 3). intracanal calcium hydroxide was resorbing. After anesthesia and rubber dam isolation (Hygienic Corporation, $\mathrm{OH}$ 44310, USA) temporary restoration was removed and intracanal calcium hydroxide was removed using irrigation and some instrumentation. Cervical third of canals were flared with sizes 1-3 Gates Glidden drills (Dentsply, Maillefer, Baillaigues, Switzerland) and prepared to full working length using Protaper universal (Dentsply, Maillefer, Baillaigues, Switzerland) rotary files. During canal preparation Protaper SX file separated in the most curved area at the apical third of mesiobuccal canal (Figure 4- Figure 5). Because of higher risk of root perforation due to thin roots, decision of bypassing the broken instrument was made [9,10]. The broken instrument was bypassed carefully by using sizes 6,8 and 10 K-files (Dentsply, Maillefer, Baillaigues, Switzerland) which were pre-curved (Figure 6). Copious irrigation was done using 5.25\% NaOCl (Septodont, Saint-Maur, France) using a side vented needle (SuYun, Jiangsu, China). Once broken file was successfully bypassed the canals were prepared in usual manner up to Protaper size F-1 in mesial canals and Protaper size F-2 in distal canal. Mesiobuccal canal was prepared manually beyond broken file up to kfile size 30 (Dentsply, Maillefer, Baillaigues, Switzerland). All canals were shaped and cleaned to full working length and irrigated using 5.25\% NaOCl. Final rinse was done using 17\% EDTA (META, Chungbuk, ROK) and canals were dried using sterile paper points (Dentsply, Maillefer, Baillaigues, Switzerland). A master cone radiograph was taken (Figure 7). Obturation was done using warm vertical compaction technique (Figure 8) to achieve good seal in all dimensions of root canal space with apical gauging of master cone gutta percha [11]. Resin based AH Plus sealer (Dentsply, Maillefer, Baillaigues, Switzerland) was used. A temporary restoration was placed and patient was scheduled for permanent full coverage cast restoration after one week.

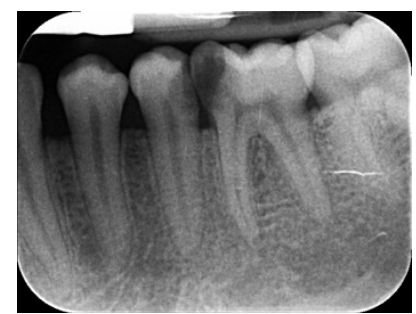

Figure 1. Preoperative diagnostic periapical X-ray

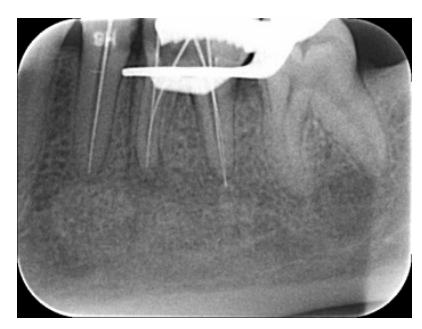

Figure 2. Initial working length X-ray

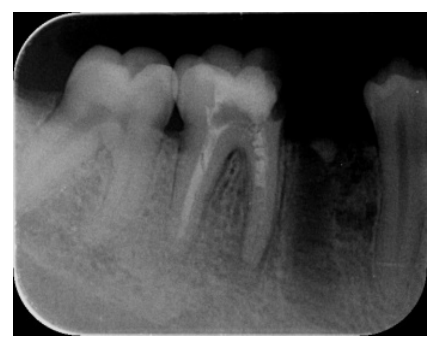

Figure 3. X-ray at second visit, note that $\mathrm{Ca}(\mathrm{OH})^{2}$ is resorbing and adjacent premolar is extracted

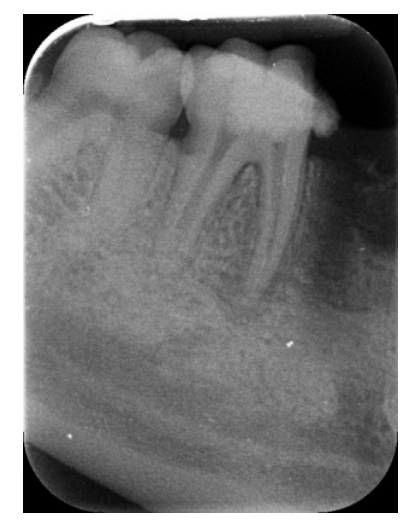

Figure 4. Broken instrument at apical 3rd in mesial root of first molar tooth

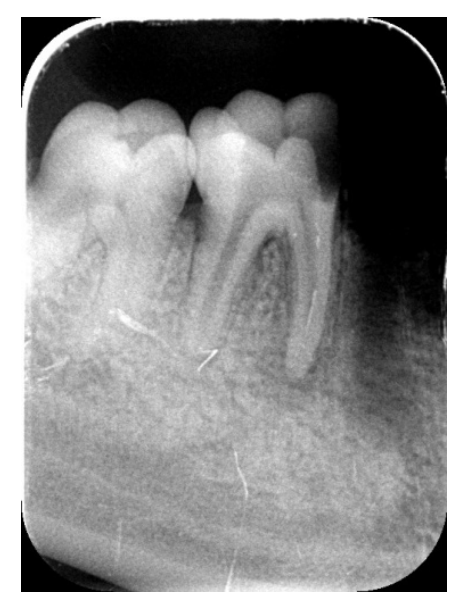

Figure 5. Broken instrument X-ray with a different angle 


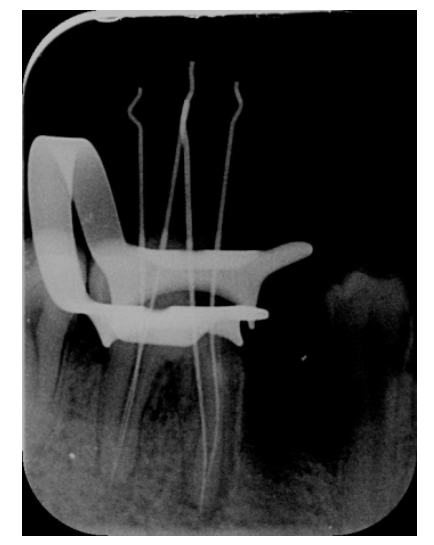

Figure 6. broken Instrument bypassed

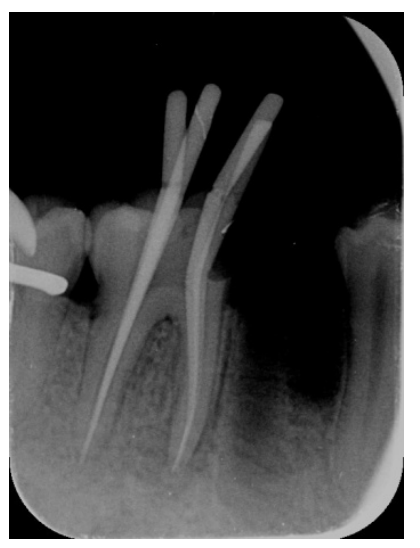

Figure 7. X-ray with master cone gutta percha

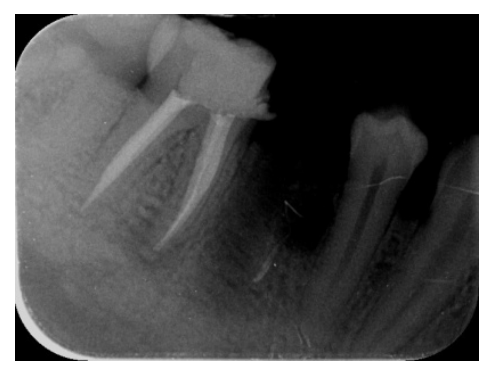

Figure 8. Final obturation with thermoplasticized injectable gutta percha technique

\section{Discussion}

Various factors contribute towards decision making in management of an intracanal broken instrument during endodontic treatment $[8,9,10]$. If proper bypassing of the instrument is done then basic objectives of root canal preparation i.e. shaping and cleaning can be achieved easily. Hence, bypassing the separated instrument is categorized as a successful approach, particularly when there are no studies carried out comparing the treatment outcome of bypassing broken instruments with their removal $[12,13,14,15,16]$. Because of potential risk of root perforation in apical third of root canal especially in thin rooted teeth, bypassing should be optimally carried out under high magnification with use of hand files and radiographic assessments to avoid such complications [9]. There are many other complications notably ledge formation, secondary separation of instruments, fragment extrusion and pushing the fragment apically that should be thought of beforehand and managed [8].

In this case because instrument was separated in the apical third of root canal where not only root canal was extremely curved but also tooth had very thin root, it was wise decision to bypass the instrument. Because of successful bypassing and shaping and cleaning of root canal beyond the separated instrument, canals were obturated successfully incorporating the broken fragment within the obturation material. Attempting to bypass the broken instrument, partially or completely, usually minimizes contact between root canal wall and broken instrument and sometimes may dislodge it. Therefore, once successfully bypassed there are considerable chances that canal space around the broken fragment and beyond the broken fragment has successfully shaped and disinfected $[9,17]$. Finally, a resin based sealer and warm vertical compaction method of obturation were used in this case so that an optimum three dimensional or nearly three-dimensional seal can be achieved for a successful treatment outcome [18,22].

\section{Conclusion}

Careful use of hand files along with radiographic checks enable the bypassing of broken instrument in the apical third of curved canals. This procedure should be considered as practical approach in teeth with thin root structures where attempts of instrument removal are associated with significant risks of root perforation or weakening of root and subsequent risk of fracture. This is a minimally invasive approach without any damage to root structure.

\section{Conflict of Interest}

The author declares there is no conflict of interest.

\section{References}

[1] Cohen S. Pathways of the pulp. 9th ed: Mosby; 2006.

[2] Spili P, Parashos P, Messer HH. The impact of instrument fracture on outcome of endodontic treatment. J Endod. 2005; 31(12): 845-50.

[3] Wolcott S, Wolcott J, Ishley D, Kennedy W, Johnson S, Minnich $\mathrm{S}$, et al. Separation incidence of protaper rotary instruments: a large cohort clinical evaluation. J Endod. 2006; 32(12): 1139-41.

[4] Iqbal MK, Kohli MR, Kim JS. A retrospective clinical study of incidence of root canal instrument separation in an endodontics graduate program: a PennEndo database study. J Endod. 2006; 32(11):1048-52.

[5] Knowles KI, Hammond NB, Biggs SG, Ibarrola JL. Incidence of instrument separation using LightSpeed rotary instruments. J Endod. 2006; 32(1): 14-6.

[6] McCoy T. Managing Endodontic Instrument Separation. J Vet Dent. 2015; 32(4): 262-5.

[7] Walia HM, Brantley WA, Gerstein H. An initial investigation of the bending and torsional properties of Nitinol root canal files. $\mathrm{J}$ Endod. 1988; 14(7): 346-51.

[8] Madarati AA, Hunter MJ, Dummer PM. Management of intracanal separated instruments. J Endod. 2013; 39(5): 569-81.

[9] Suter B, Lussi A, Sequeira P. Probability of removing fractured instruments from root canals. Int Endod J. 2005; 38(2): 112-23. 
[10] Madarati AA, Watts DC, Qualtrough AJ. Opinions and attitudes of endodontists and general dental practitioners in the UK towards the intracanal fracture of endodontic instruments: part 1. Int Endod J. 2008; 41(8): 693-701.

[11] Lally TT, Pacheco E, Bey GL. Creating Well-Obturated Canals. The Use of Warm Vertical Condensation Techniques. Dent Today. 2016; 35(4): 76-8, 80.

[12] Hulsmann M, Schinkel I. Influence of several factors on the success or failure of removal of fractured instruments from the root canal. Endodontics \& dental traumatology. 1999; 15(6): 252-8.

[13] Al-Fouzan KS. Incidence of rotary ProFile instrument fracture and the potential for bypassing in vivo. Int Endod J. 2003; 36(12): 864-7.

[14] Shen Y, Peng B, Cheung GS. Factors associated with the removal of fractured NiTi instruments from root canal systems. Oral Surg Oral Med Oral Pathol Oral Radiol Endod. 2004; 98(5): 605-10.

[15] Parashos P, Messer HH. Rotary NiTi instrument fracture and its consequences. J Endod. 2006; 32(11): 1031-43.

[16] Nevares G, Cunha RS, Zuolo ML, Bueno CE. Success rates for removing or bypassing fractured instruments: a prospective clinical study. J Endod. 2012; 38(4): 442-4.
[17] Cuje J, Bargholz C, Hulsmann M. The outcome of retained instrument removal in a specialist practice. Int Endod J. 2010; 43(7): 545-54

[18] Aminsobhani M, Ghorbanzadeh A, Sharifian MR, Namjou S, Kharazifard MJ. Comparison of Obturation Quality in Modified Continuous Wave Compaction, Continuous Wave Compaction, Lateral Compaction and Warm Vertical Compaction Techniques. J Dent (Tehran). 2015; 12(2): 99-108.

[19] Aqrabawi JA. Outcome of endodontic treatment of teeth filled using lateral condensation versus vertical compaction (Schilder's technique). J Contemp Dent Pract. 2006; 7(1): 17-24.

[20] Chen Z. [Difficulties and misunderstandings of root canal filling]. Hua Xi Kou Qiang Yi Xue Za Zhi. 2017; 35(3): 232-8.

[21] Farzaneh M, Abitbol S, Lawrence HP, Friedman S, Toronto S. Treatment outcome in endodontics-the Toronto Study. Phase II: initial treatment. J Endod. 2004; 30(5): 302-9.

[22] Fleming CH, Litaker MS, Alley LW, Eleazer PD. Comparison of classic endodontic techniques versus contemporary techniques on endodontic treatment success. J Endod. 2010; 36(3): 414-8. 